\title{
A COMPARISON OF TWO- AND THREE-VARIABLE MODELS FOR COMBUSTION IN SEALED CONTAINERS
}

\author{
CLAIRE E. TRENHAM ${ }^{\Perp 1}$ and LARRY K. FORBES 1
}

(Received 3 March, 2005; revised 9 January, 2006)

\begin{abstract}
This paper analyses a model for combustion of a self-heating chemical (such as pool chlorine), stored in drums within a shipping container. The system is described by three coupled nonlinear differential equations for the concentration of the chemical, its temperature and the temperature within the shipping container. Self-sustained oscillations are found to occur, as a result of Hopf bifurcation. Temperature and concentration profiles are presented and compared with the predictions of a simpler two-variable approximation for the system. We study the period of oscillation and its variation with respect to the ambient temperature and the reaction parameter. Nonlinear resonances are found to exist, as the solution jumps between branches having different periods.
\end{abstract}

2000 Mathematics subject classification: primary $80 \mathrm{~A} 25$; secondary $34 \mathrm{Cl}, 70 \mathrm{~K} 30$, $70 \mathrm{~K} 50$.

Keywords and phrases: combustion models, limit cycles, nonlinear resonances, relaxation oscillations.

\section{Introduction}

This paper studies the problem describing the thermodynamic effects that occur when isolated sealed drums of a chemical, such as pool chlorine, are stored in a larger shipping container. Each drum contains material that is capable of self-heating as it decays (exothermic decay), and this in turn can heat the air within the container by Newtonian energy transfer. Thus the sealed drums are able to interact with one another thermally, although they are chemically isolated. In a recent (2001) paper, Gray [12] considered each drum to be "well-stirred", so that the temperature in the drum is constant throughout its volume. That is, each drum is the equivalent of a unit of variable heat, and Gray thus adopted the term "thermon" to describe it.

\footnotetext{
'School of Mathematics and Physics, University of Tasmania, G.P.O. Box 252-37, Hobart 7001 TAS, Australia; e-mail: ctrenham@utas.edu.au.

(C) Australian Mathematical Society 2006, Serial-fee code 1446-181 1/06
} 
In Gray's model, the "pool chemical" approximation was made for both steps in the reaction [26], as a result of which the consumption of all chemicals was ignored. The "thermons" were housed within a larger container (or shipping container), in which the air temperature could vary also. The larger container could undergo Newtonian cooling to ambient. Each region was approximated as "well-stirred", so that the temperatures were described by a system of nonlinear ordinary differential equations (ODEs). Gray [12] found the presence of saddle-node bifurcations, which may give the possibility of explosion in the physical system. He showed that Hopf bifurcation $[2,19]$ did not occur in the system, and conjectured that no self-sustained oscillations would be possible.

Gray [12] analysed his model in detail for the case in which all the drums (thermons) were identical, since in this case the model reduced to a system of two ODEs. Such a dynamical-systems approach allowed him to identify parameter regions in which spontaneous combustion could occur, and to estimate the time to ignition.

There is a large amount of literature on the use of dynamical-systems techniques in physical chemistry, and much of this is discussed in the book by Scott [26]. One of the simplest models of temperature-sensitive reactions is the Sal'nikov scheme [24,25], which involves only two nonlinear ODEs, for the concentration and temperature of a particular chemical species in a two-step reaction $[6,10,13-15,22]$. The reaction may proceed via either one or two exothermic decay steps, and may be considered to be in a drum heating a surrounding, possibly enclosed area, or a series of such drums $[4,12]$. A key feature of such a scheme is that one or more of the chemical reactions involved has a reaction rate that varies with temperature, according to Arrhenius kinetics $[3,5,11,16,20]$. The Sal'nikov scheme is typically found to induce chemical oscillations $[17,21]$ resulting from Hopf bifurcation. Other systems studied in physical chemistry include burning models [7,8], the Belousov-Zhabotinskii reaction [27] and semibatch reactors $[22,28]$, forced systems [9] where an extra forcing term is included to modulate oscillations, and a wide range of phase and biological reactions as discussed by Scott [26].

The present paper extends Gray's model, by allowing the effects of chemical consumption to be included. This adds an extra unknown parameter to the model, and an extra ODE (rate equation) to the system for each drum present. We retain the pool chemical approximation for the first stage of the two-step reaction, but allow for consumption of the intermediate reagent. Only the second step of the reaction is exothermic in this study. A quasi-stationary approximation to this system yields a Sal'nikov reaction. The predictions of this model are then compared with the three-variable system without approximation.

The model is derived in Section 2, and the two-variable approximation is developed in Section 3. An analysis of the full three-dimensional model is then presented in Section 4 , and the results of numerical calculations given in Section 5. A discussion 
of the results in Section 6 concludes the paper.

\section{The governing model}

Consider a precursor chemical species (reagent) $A$, that decays to form an intermediate $X$ and then a final inert product $B$. Each reaction is assumed to follow first-order kinetics, so that

$$
A \stackrel{k_{0}}{\rightarrow} X \stackrel{k_{1}}{\rightarrow} B
$$

Species $A$ is assumed to be in vast over-supply, so that its molar concentration [ $A]$ does not vary appreciably over time. This is the "pool-chemical approximation" [26]. The first reaction in the scheme (2.1) is assumed to progress at the constant rate $k_{0}$, but the second reaction in (2.1) is taken to have the temperature-sensitive rate

$$
k_{1}(T)=Z e^{-E / R T},
$$

according to Arrhenius kinetics [3]. The symbol $Z$ is the reference rate, and $E$ and $R$ denote the activation energy for the reaction and the universal gas constant respectively.

The rate equation and conservation of energy for drum number $j$ in a system of $n$ drums then leads to the system of ODEs

$$
\begin{aligned}
\frac{d\left[X_{j}\right]}{d t} & =k_{j 0}\left[A_{j}\right]-k_{j 1}\left(T_{j}\right)\left[X_{j}\right], \\
\rho_{j} c_{j} V_{j} \frac{d T_{j}}{d t} & =Q_{j} m_{j}^{W} V_{j}\left[X_{j}\right] k_{j 1}\left(T_{j}\right)+\chi_{j} S_{j}\left(T_{D}-T_{j}\right), \quad j=1, \ldots, n .
\end{aligned}
$$

This is supplemented by conservation of energy for the entire container, to give

$$
\rho_{D} c_{D} V_{D} \frac{d T_{D}}{d t}=\sum_{j=1}^{n} \chi_{j} S_{j}\left(T_{j}-T_{D}\right)-\chi_{D} S_{D}\left(T_{D}-T_{a}\right) .
$$

Here $k_{j 0}$ represents the rate of the constant step for each drum, with $k_{j 1}\left(T_{j}\right)$ the exothermic rate, given in Equation (2.2). The specific heat of the material in each drum is $c_{j}$, the density $\rho_{j}$ and the molecular weight $m_{j}^{w}$. The concentration of the pool chemical, $A$, is $\left[A_{j}\right]$, with $Q_{j}$ the exothermic heating term and $\chi_{j}$ is a Newtonian cooling coefficient representing energy/degree/area/time. The volume of each drum is $V_{j}$ with surface area $S_{j}$.

This differs from Gray's (2001) [12] "thermon" model only in the inclusion of the extra rate equations (2.3) for the consumption of the chemical species in each drum.

Dimensionless variables are now introduced, and will be used throughout the rest of the paper. These amended variables are merely scales of the variables given above 
with respect to a constant sharing those units. In the process a few variables can be combined and renamed to simplify the problem. Let all times be scaled with respect to a reference time,

$$
\tau_{s}=\frac{\rho_{1} c_{1} E_{1}}{Q_{1} m_{1}^{W}\left[A_{1}\right] Z_{1} R} .
$$

Temperatures are made dimensionless with respect to the quantity $R / E_{1}$, and the concentrations are referenced to the constant $\left[A_{1}\right]$. In addition it will be assumed here that each drum is identical, so that $j=1$ only, in Equations (2.3) and (2.4).

The model may then be seen to depend upon six nondimensional parameters,

$$
\begin{aligned}
& \alpha=\frac{\rho_{D} c_{D} V_{D}}{\rho_{1} c_{1} V_{1}}, \quad \beta_{D}=\frac{\chi_{D} S_{D}}{\chi_{1} S_{1}}, \quad \quad \mu_{1}=k_{10} \tau_{s}, \\
& \theta_{a}=\frac{R T_{a}}{E_{1}}, \quad \lambda_{1}=\frac{\chi_{1} S_{1} E_{1}}{V_{1} Q_{1} m_{1}^{W}\left[A_{1}\right] Z_{1} R} \quad \text { and } n .
\end{aligned}
$$

Thus we obtain the governing system of equations in dimensionless form, for the case in which all drums in the container are assumed to be identical. The system is

$$
\begin{aligned}
\frac{d X_{1}}{d t} & =\mu_{1}-X_{1} e^{-1 / T_{1}}, \\
\frac{d T_{1}}{d t} & =X_{1} e^{-1 / T_{1}}+\lambda_{1}\left(T_{D}-T_{1}\right), \\
\alpha \frac{d T_{D}}{d t} & =n \lambda_{1}\left(T_{1}-T_{D}\right)-\beta_{D} \lambda_{1}\left(T_{D}-\theta_{a}\right) .
\end{aligned}
$$

The first parameter in (2.5) effectively represents a volume and specific heat ratio between the container and any of the drums. The second is a surface area ratio between the container and the drums. The third represents a nondimensional rate term dependent on the reaction. The scaled ambient temperature, $\theta_{a}$, is a nondimensional temperature of the environment outside the container, with $\lambda_{1}$ the heat transfer coefficient between the drums and the container. Finally, $n$ is the number of drums in the container, as in Gray [12].

\section{Two-variable quasi-stationary approximation}

In this section we present a simplified analysis of the system (2.6)-(2.8), based on a quasi-equilibrium assumption for $T_{D}$. This was originally suggested by an analysis of the numerical solutions of the full system (2.6)-(2.8).

In this case, Equation $(2.8)$ becomes $0 \approx n \lambda_{1}\left(T_{1}-T_{D}\right)-\beta_{D} \lambda_{1}\left(T_{D}-\theta_{a}\right)$. This leads at once to the approximate relation

$$
T_{D} \approx\left(\frac{n}{n+\beta_{D}}\right) T_{1}+\frac{\beta_{D} \theta_{a}}{n+\beta_{D}}
$$


This result is now substituted into Equations (2.6) and (2.7) to give the approximate phase-plane system

$$
\begin{aligned}
\frac{d X_{1}}{d t} & =\mu_{1}-X_{1} e^{-1 / T_{1}}, \\
\frac{d T_{1}}{d t} & =X_{1} e^{-1 / T_{1}}-\frac{\beta_{D} \lambda_{1}}{n+\beta_{D}}\left(T_{1}-\theta_{a}\right) .
\end{aligned}
$$

This is a Sal'nikov system $[24,25]$, and so its behaviour can be analysed extensively in the $\left(X_{1}, T_{1}\right)$ phase plane.

Equilibrium occurs in the system when both rates of change are equal to zero. This occurs at the point

$$
\left(X_{1 e}, T_{1 e}\right)=\left(\mu_{1} \exp \left(\frac{\beta_{D} \lambda_{1}}{\mu_{1}\left(n+\beta_{D}\right)+\theta_{a} \beta_{D} \lambda_{1}}\right), \frac{\mu_{1}\left(n+\beta_{D}\right)}{\beta_{D} \lambda_{1}}+\theta_{a}\right) .
$$

To determine the local solution behaviour near this point, it is necessary to linearise the system about the point using the Hartman-Grobman linearisation theorem [18]. This requires a calculation of the eigenvalues of the $(2 \times 2)$ Jacobian matrix of partial derivatives for the system, evaluated at the equilibrium point (3.3). The eigenvalues $\xi$ are found by solving

$$
\operatorname{det}|J-I \xi|=\xi^{2}-T_{J} \xi+D_{J}=0,
$$

in which $T_{J}$ and $D_{J}$ denote the trace and determinant of the Jacobian matrix, respectively.

The eigenvalues are clearly given by the formula

$$
\xi=\frac{1}{2}\left(T_{J} \pm \sqrt{T_{J}^{2}-4 D_{J}}\right) .
$$

When $D_{J}>0$ and $T_{J}^{2}<4 D_{J}$ then the stability of the equilibrium point (3.3) is determined by the sign of the trace $T_{J}$. A stable focus occurs for $T_{J}<0$, and an unstable focus is formed if $T_{J}>0$.

Hopf bifurcation may occur in system (3.1)-(3.2) when $T_{J}=0$ and $D_{J}>0$, as this is the point at which stability changes. The conditions required for a saddle-node bifurcation are found never to occur for physical values of the parameters in this system where we include chemical consumption. The above analysis leads to the Hopf condition

$$
\exp \left(\frac{-\beta_{D} \lambda_{1}}{\mu_{1}\left(n+\beta_{D}\right)+\theta_{a} \beta_{D} \lambda_{1}}\right)-\frac{\mu_{1}\left(\beta_{D} \lambda_{1}\right)^{2}}{\left(\mu_{1}\left(n+\beta_{D}\right)+\theta_{a} \beta_{D} \lambda_{1}\right)^{2}}+\frac{\beta_{D} \lambda_{1}}{n+\beta_{D}}=0 .
$$

This equation describing the condition for Hopf bifurcation is transcendental, but can be solved numerically. 


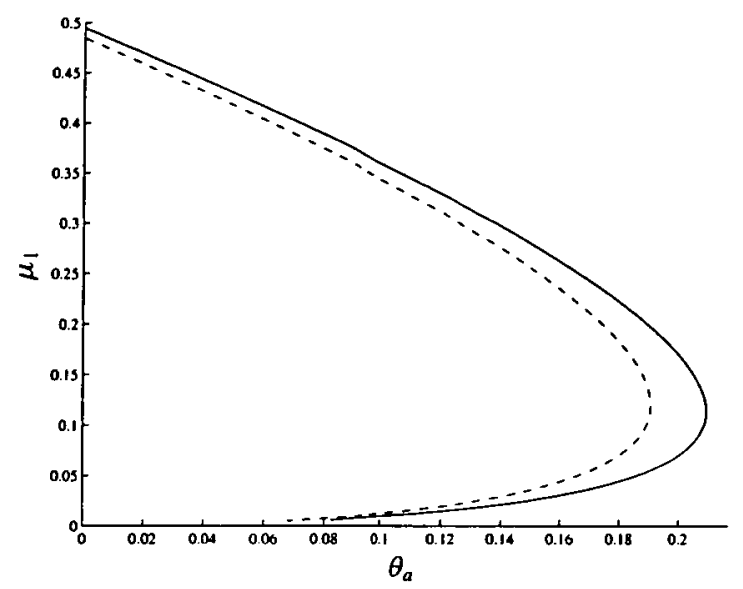

FIGURE 1. Hopf curves for the two systems. The dashed line represents the Hopf curve of the 2D system with the solid line representing the $3 \mathrm{D}$ system. Both use the parameters $\lambda_{1}=0.9, \alpha=27, \beta_{D}=9$ and $n=2$.

The dashed curve in Figure 1 is a Hopf curve obtained from (3.4), for the case $\lambda_{1}=0.9, \beta_{D}=9$ and $n=2$. This curve is typical of other values of $\lambda_{1}$ and $\beta_{D}$, which change the curve little within a reasonable range of each variable. However as $n$ increases, the curve becomes thinner and thinner (the top limb moves downward, the lower limb is near-stationary) until as $n \rightarrow \infty$ the curve disappears altogether. The equilibrium point (3.3) is stable to the right of this dashed line, but is unstable $\left(T_{J}>0\right)$ to the left. Limit cycle oscillations are born on the Hopf curve, and stable finite-amplitude oscillatory behaviour is encountered for parameter values to the left of this curve.

Figure 2 (b), seen in Section 5, shows a limit cycle computed by the numerical solution of Equations (3.1) and (3.2), for the same parameter values as in Figure 1, and for $\theta_{a}=0.11, \mu_{1}=0.08$. This represents a point well to the left of the dashed Hopf curve. Figure 2 (c) shows the variation of the concentration of the intermediate chemical $X$ with respect to time, $t$, with a dashed line. Note the variation shows relaxation oscillation behaviour. Figure 2 (d) shows the variation of the temperature of any given drum in the container $\left(T_{1}\right)$ with respect to time $(t)$. In this case the periodicity of the variation is clear, and the departure from sinusoidal behaviour indicates pronounced effects of nonlinearity.

In this case it is possible to prove the nonexistence of limit cycles for some parameter values outside the Hopf curve (to the right of the dashed line in Figure 1), using the Dulac theorem [23]. This involves a similar process to that outlined by Forbes et al. [10] for a Sal'nikov scheme exothermic in both steps, and again indicates that there exist no limit cycles for $\theta_{a}>1 / 4$. 


\section{Analysis of the three-variable system}

In this section we give an analysis of the three-dimensional system derived in Section 1, that is, Equations (2.6)-(2.8). This is not a Sal'nikov system, but can be approximated to one as in Section 2.

This system has only one equilibrium point, occurring when

$$
\begin{aligned}
& \left(X_{\text {le }}, T_{1 e}, T_{D e}\right) \\
& \quad=\left(\mu_{1} \exp \left(\frac{\beta_{D} \lambda_{1}}{\mu_{1}\left(n+\beta_{D}\right)+\beta_{D} \lambda_{1} \theta_{a}}\right), \frac{\mu_{1}\left(n+\beta_{D}\right)}{\beta_{D} \lambda_{1}}+\theta_{a}, \frac{n \mu_{1}}{\beta_{D} \lambda_{1}}+\theta_{a}\right) .
\end{aligned}
$$

Again, the system is linearised about this point by the Hartman-Grobman theorem, using the Jacobian matrix, which is now a $3 \times 3$ matrix of partial derivatives. The eigenvalues $\xi$ of the Jacobian matrix satisfy a cubic equation [1] of the form

$$
\xi^{3}+a_{2} \xi^{2}+a_{1} \xi+a_{0}=0 .
$$

The constants $a_{0}, a_{1}$ and $a_{2}$ are known functions of the six dimensionless parameters in Equation (2.5). The equilibrium point (4.1) will be stable if all eigenvalues $\xi$ have negative real parts, and unstable otherwise.

Hopf bifurcation occurs when any pair of eigenvalues crosses the imaginary axis, at points $\xi= \pm i \gamma$. This will occur if

$$
\xi^{3}+a_{2} \xi^{2}+a_{1} \xi+a_{0}=\left(\xi-\xi_{0}\right)(\xi+i \gamma)(\xi-i \gamma)=0
$$

for $\xi_{0}$ and $\gamma$ real. The Hopf condition for the creation of limit cycles in this threevariable system is thus

$$
a_{0}=a_{1} a_{2}, \quad a_{1}>0 .
$$

Numerical solution of the system for these conditions results in a curve of Hopf bifurcations in the $\left(\mu_{1}, \theta_{a}\right)$ plane similar to that for the simplified system, and is now shown in Figure 1 by a solid line. Here the same parameter values were used as for the quasi-stationary case, and with $\alpha=27$. As in the quasi-stationary case, the equilibrium point (4.1) is stable to the right of this solid line and unstable to the left. Again, limit cycles are born on the Hopf curve, and exist as finite-amplitude orbits in phase space to the left of the line.

\section{Presentation of results}

Detailed solutions have been computed using MATLAB ${ }^{\circledR}$, and it is found that the Hopf bifurcation produces stable limit cycles. An example of this is given in Figure 2 (a), for the reaction parameter $\mu_{1}=0.08$ and ambient temperature $\theta_{a}=0.11$, a 


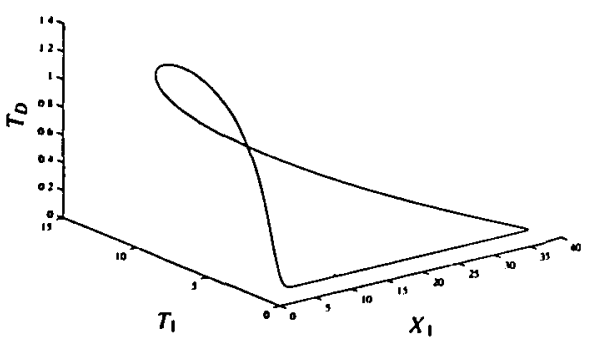

(a)

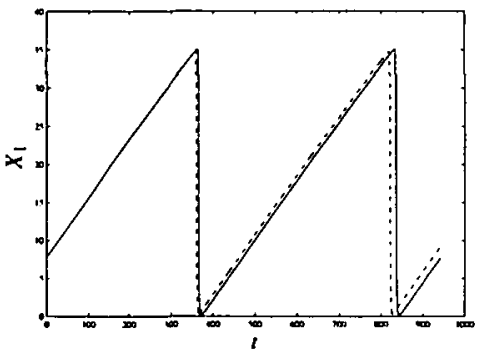

(c)

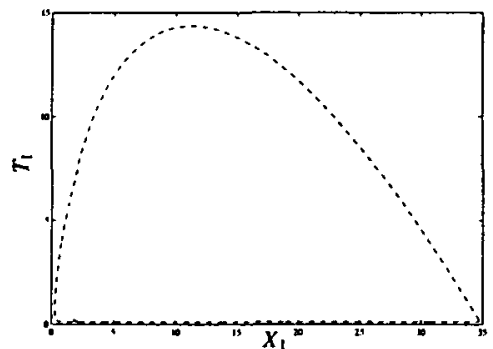

(b)

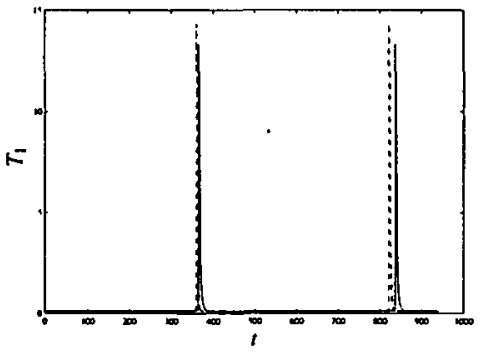

(d)

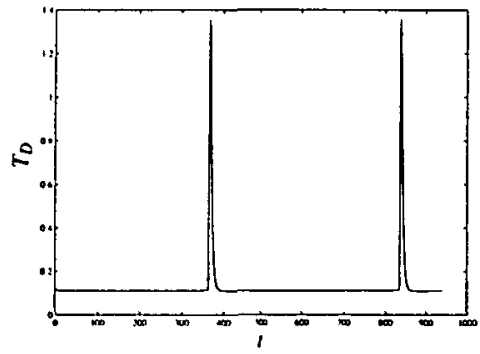

(e)

FIGURE 2. Throughout this plot, the quasi-stationary (2D) case is represented by a dashed line, with the full three-dimensional system being drawn with a solid line. (a) A sample limit cycle in the 3D case, with $\mu_{1}=0.08$ and $\theta_{a}=0.11$. (b) A limit cycle in the quasi-stationary approximation (2D case), with the same coordinates as in the $3 \mathrm{D}$ case. The sharp corner at the right-hand side of this plot suggests the extreme changes present in relaxation oscillations. (c) Plot of concentration versus time for the limit cycles, showing a gradual build-up of $X_{1}$ over time followed by a very fast dissipation. During $X_{1}$ build up, both temperatures remain constant, then with the sudden relaxation in concentration we see instantaneous spikes, multiplying one hundred-fold in $T_{1}$ and ten-fold in $T_{D}$, as seen in parts (d) and (e) respectively. (d) Equivalent plot of drum temperature versus time for the limit cycles, showing emphasised, sudden peaks in both cases. which implies that the 2D system gives a good approximation to the full 3D system. (e) Plot of the container temperature, $T_{D}$, over time. This mirrors the behaviour of the drum temperature. Only the $3 \mathrm{D}$ plot is shown as the $2 \mathrm{D}$ plot effectively assumes an infinitely large container, thus $T_{D}=\theta_{a}=$ constant. 


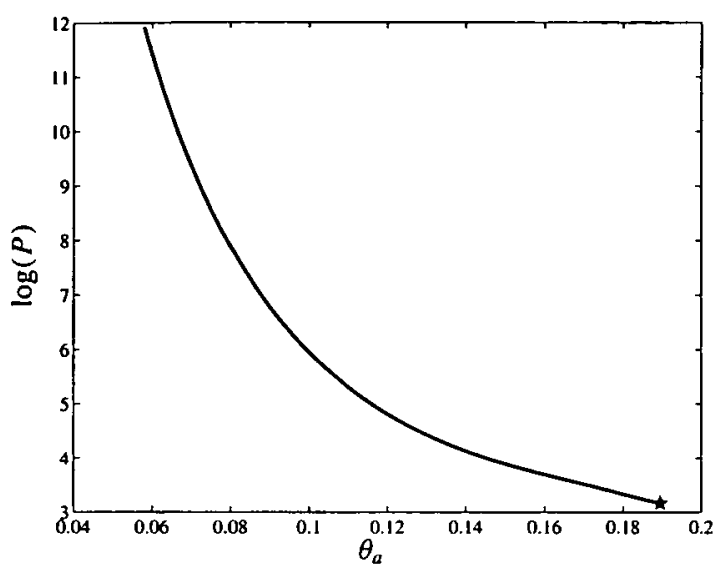

FIGURE 3. Dependence of oscillation period on $\theta_{a}$, the ambient temperature. The star marks the period predicted by the eigenvalues at the Hopf point. Note that the vertical scale is logarithmic due to the very large increases in period with small decreases in ambient temperature.

point well inside the Hopf curve. Figure 2 (c) shows the variation of concentration of chemical $X$ in a drum with respect to time for this same case, and exhibits saw-tooth behaviour typical of relaxation oscillations. Figures 2 (d) and (e) show the variation of the drum temperature $T_{1}$ and shipping container temperature $T_{D}$ respectively over time. Figures 2 (c)-(e) show clear evidence of relaxation oscillation behaviour, in which the concentration of $X_{1}$ exhibits slow rises alternating with abrupt, almost discontinuous, falls. The two temperature profiles possess dramatic spikes at the times when the concentration drops suddenly. These sudden temperature excursions may in practice give rise to spontaneous explosion. We can see comparing the solid (3D) and dashed (2D) lines in the plots that the quasi-stationary approximation to the full system is a fairly good approximation, but it slightly underestimates the period and overestimates the magnitude of the temperature spikes.

We wish to judge the period of this relaxation oscillation in the three-dimensional case (as this is the more accurate case), and thus its severity (as the greater the period, the more extreme the relaxation oscillation and the greater the temperature spike). Thus the shooting method employed by Forbes is used $[6,7,9,10]$, and a plot made of the period of the oscillation with respect to the ambient temperature of the system, shown in Figure 3, and also of the period with respect to the reaction-dependent parameter $\mu_{1}$, shown in Figure 4 . It can be seen, as one would expect, that as the period of the limit cycle increases with varying $\theta_{a}$, the spike in the drum and container temperatures also increases, however the ratio of the three quantities remains the same. When the parameter $\mu_{1}$ is varied, the temperature spikes remain virtually constant, increasing slowly toward the top limb of the Hopf curve. 


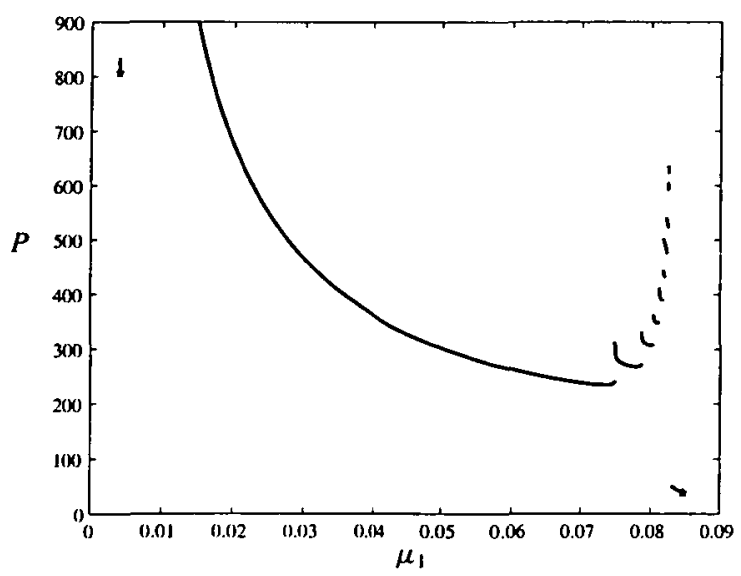

FIGURE 4. Dependence of period on the reaction coefficient, $\mu_{1}$. Note the resonance structure occurring as $\mu_{1}$ increases toward the upper limb of the Hopf curve. Here $\lambda_{1}=0.3$ and $\theta_{a}=0.11$. Stars mark the period of the oscillation as predicted by the eigenvalues at the Hopf points. The discontinuity arises from the onset of small secondary loops contained within the large limit cycle. The period of the large limit cycle continues to grow rapidly below the point of the lower Hopf curve, suggesting a subcritical Hopf bifurcation at that point and a subsequent fold bifurcation.

At the points of Hopf bifurcation, the period $P$ of oscillation can be predicted from the eigenvalues of the Jacobi matrix, using the formula

$$
P=\frac{2 \pi}{\Im(\xi)} \text {. }
$$

The eigenvalues are numerically calculated and their imaginary component substituted into (5.1) to find the Hopf-point periods, and this theoretical value is marked using stars in Figures 3 and 4.

Figure 3 shows the dependence of the period of the limit cycle (for the full threedimensional system, still assuming all barrels are identical), upon the ambient temperature $\theta_{a}$ of the system, for the value $\mu_{1}=0.2$ (that is, a horizontal cut through Figure 1, with $\lambda_{1}=0.9, \beta_{D}=9, \alpha=27$ and $n=2$ ). This period is predicted at the point of Hopf bifurcation by Equation (5.1), and this predicted period is shown with a star at the far right-hand side of the figure. The plot exhibits a rapid increase in period $P$ as the ambient temperature of the system, $\theta_{a}$ decreases. The rate of increase of period $P$ is emphasised by noting that the scale of the vertical axis of this graph is actually logarithmic rather than linear. As the period of the limit cycle increases, the relaxation component of the oscillation becomes more pronounced; that is, the size of the spikes in Figures 2 (c) and (d) increases with decreasing ambient temperature $\theta_{a}$ in Figure 3. Such extended spikes reflect in larger and more sudden explosions in the system as the spikes become more pronounced. 
Figure 4 shows the dependence of the period of the oscillation upon the reaction parameter $\mu_{1}$, for $\lambda_{1}=0.3$ and $\theta_{a}=0.11$. In this case, the Hopf curve has the same shape as in Figure 1, but intersects the vertical axis at a lower value of $\mu_{1}$. Figure 4 effectively represents a vertical cut through that Hopf curve, and again, for each Hopf point (as there are two Hopf points vertically), the period is predicted from (5.1) and shown by a star. This plot is interesting in form, but was only found to occur thus for low values of $\lambda_{1}$.

Near each of the two Hopf points, it was possible to compute numerical solutions that continue the period of the cycle on a connected branch. These are shown in Figure 4. However it is clear on the right-hand side of the figure that there are numerous disconnected nonlinear solution branches, in the approximate interval

$$
0.07485<\mu_{1}<0.0825 \text {. }
$$

These are nonlinear resonances in the limit cycle itself, where the period of oscillation jumps discontinuously. We have computed approximately ten of these disconnected solution branches, each of which is characterised by a longer period than the last, as is evident in Figure 4. The limit cycles on successive branches (increasing $\mu_{1}$ ) exist for narrower and narrower intervals in the reaction parameter $\mu_{1}$, and develop very long periods. For this reason, they become increasingly difficult to isolate. It is even possible that chaos may exist in a narrow parameter window around $\mu_{1}=0.0828$, in which the period would become infinite. For larger values of the heat transfer coefficient, such as $\lambda_{1}=0.9$ as used earlier in this paper, we do not see these extreme resonance structures. We are able to find the first resonance branch, but nothing beyond this. Thus we hypothesise that this resonance behaviour is typical of the system but only detectable for extreme values of $\lambda_{1}$.

We now consider the second Hopf point, indicated by a star on the left of Figure 4. In this case it has been found that limit cycles could be computed in a narrow window of $\mu_{1}$ values below the Hopf value. This strongly suggests that the limit cycle branch may undergo a fold bifurcation at a value of $\mu_{1}$ below the Hopf value, before reconnecting to the Hopf point. Such a structure would involve the existence of unstable limit cycles, and possibly even a subcritical Hopf bifurcation at the lower Hopf point. The rapid growth of the period of the limit cycle in the parameter region to the right of the lower Hopf point suggests that a second band of chaos may also be present in this region, as indicated by computing the Floquet multipliers for the system.

Whilst it would appear that multiple limit cycles may exist for some values of $\mu_{1}$, the authors were unable to find any instance of any such behaviour, despite exhaustive searching.

Figure 5 shows the qualitative change in the limit cycle, across the first resonance jump on the right-hand side of Figure 4. The limit cycle in three-dimensional phase space is shown for $\mu_{1}=0.05$ in Figure 5 (a), and for $\mu_{1}=0.075$ in Figure 5 (b). 


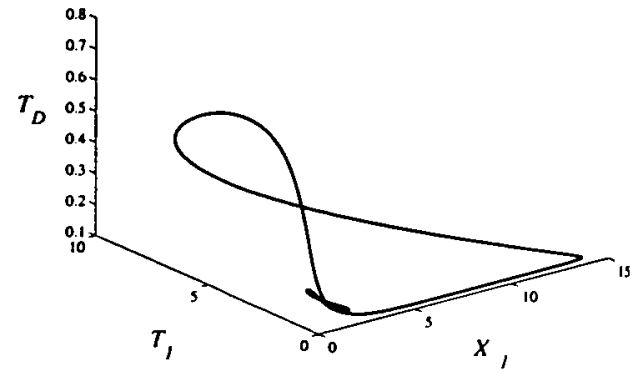

(a)

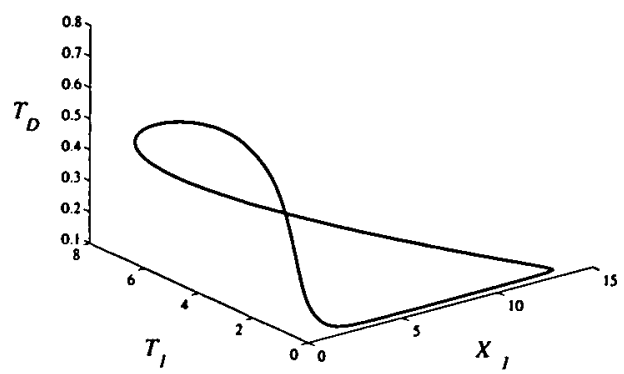

(b)

FIGURE 5. A comparison between limit cycles in the first and second resonance branches, showing the small secondary loop which, at the point of Hopf bifurcation, exists alone without the larger cycle. Here we use $\mu_{1}$ values of (a) $\mu_{1}=0.05$ for the limit cycle of the first resonance, and (b) $\mu_{1}=0.075$ for the limit cycle of the second resonance.

It is clear that the second resonance branch in Figure 5 (b) is characterised by the emergence of a smaller secondary loop in the orbit. Higher resonance branches on the right-hand side of Figure 4 appear to involve a limit cycle of similar overall shape to the orbit shown in Figure 5 (b), but possessing extra numbers of excursions around the small loop. The final low-period branch on the far right of Figure 4 appears to consist solely of this loop.

\section{Conclusion}

In this paper we have extended Gray's 2001 model [12], to include consumption of the initial chemical. In the original Gray "thermon" model, the possibility of limit cycle behaviour produced by Hopf bifurcation did not exist, and there a saddle-node bifurcation was encountered. We have derived a system describing a two-step chemical reaction in which the first step is constant and the second exothermic, for a chemical stored in drums within a shipping container. Each drum has a constant temperature throughout, and is considered to be a "thermon" in the notation of Gray [12]. The container also has a constant temperature throughout. The shipping container is surrounded by an ambient atmosphere of unchanging temperature. In contrast to Gray's model, when consumption of the intermediate reagent is included in the model, we find no saddle-node bifurcations exist, but Hopf bifurcations lead to the limit cycle oscillations found in this paper.

In Section 2 we make a quasi-stationary approximation which reduces the system to a Sal'nikov scheme, which is well understood and well studied. We present a 
brief analysis, showing a limit cycle in Figure 2 indicative of a supercritical Hopf bifurcation.

In Section 3 we analyse the system in which all drums in the shipping container start with the same temperature and behave identically. In this case we again find Hopf bifurcations. We find that the period of the limit cycle is critically dependent on the ambient temperature, with the period increasing rapidly as the ambient temperature decreases (Figure 3).

Significantly, in a chemical system which can easily be approximated by a Sal'nikov scheme, we find resonance structures occurring within the limit cycles themselves, and are able to track these for some significant range of $\mu_{1}$ values. At the edge of these resonance structures there appears to exist a fine band of chaos. Near the Hopf points (points of intersection with the Hopf curve), the period is nevertheless seen to match that predicted by the eigenvalues of the linearised analysis at the Hopf point.

\section{References}

[1] M. Abramowitz and I. A. Stegun (eds.), Handbook of Mathematical Functions (Dover, New York, 1972).

[2] L. Arnold, N.S. Namachchivaya and K. R. Schenk-Hoppe, "Toward an understanding of stochastic Hopf bifurcation: A case study", Internat. J. Bifur. Chaos Appl. Sci. Engng 6 (1996) 1947-1975.

[3] P. W. Atkins, The Elements of Physical Chemistry, 3rd ed. (Oxford University Press, Oxford, 2000).

[4] P. C. Bowes, Self heating: evaluating and controlling the hazards (H. M. Stationery Office, 1984).

[5] K. H. Ebert, P. Deuflhard and W. Jäger (eds.), Modelling of Chemical Reaction Systems, Springer Series in Chemical Physics 18, Ch. 12 and others, (Springer, Berlin, 1981).

[6] L. K. Forbes, "Limit-cycle behaviour in a model chemical reaction: the Sal'nikov thermokinetic oscillator", Proc. Roy. Soc. Lond. Ser. A Math. Phys. Eng. Sci. 430 (1990) 641-651.

[7] L. K. Forbes, "One-dimensional pattern formation in a model of burning", J. Austral. Math. Soc. Ser. $B 35$ (1993) 145-173.

[8] L. K. Forbes, "Stationary circular target patterns in a surface burning reaction", J. Engng Math. 30 (1996) $471-486$.

[9] L. K. Forbes and B. F. Gray, "Forced oscillations in an exothermic chemical reaction", Dyn. Stab. Syst. 9 (1994) 253-269.

[10] L. K. Forbes, M. R. Myerscough and B. F. Gray, "On the presence of limit-cycles in a model exothermic chemical reaction: Sal'nikov's oscillator with two temperature-dependent reaction rates", Proc. Roy. Soc. Lond. Ser. A Math. Phys. Eng. Sci. 435 (1991) 591-604.

[11] S. Glasstone, K. J. Laidler and H. Eyring, The Theory of Rate Processes, 1st ed. (McGraw-Hill, New York, 1941).

[12] B. F. Gray, "On the critical conditions for an assembly of interacting thermons", ANZIAM J. 43 (2001) $1-11$.

[13] B. F. Gray and L. K. Forbes, "Analysis of chemical kinetic systems over the entire parameter space. IV. The Sal'nikov oscillator with two temperature-dependent reaction rates", Proc. Roy. Soc. Lond. Ser. A Math. Phys. Eng. Sci. 444 (1994) 621-642. 
[14] B. F. Gray and M. J. Roberts, "Analysis of chemical kinetic systems over the entire parameter space. I. The Sal'nikov thermokinetic oscillator", Proc. Roy. Soc. Lond. Ser. A Math. Phys. Eng. Sci. 416 (1988) 391-402.

[15] B. F Gray and M. J. Roberts, "An asymptotic analysis of the Sal'nikov thermokinetic oscillator", Proc. Roy. Soc. Lond. Ser. A Math. Phys. Eng. Sci. 416 (1988) 425-441.

[16] P. Gray, S. R. Kay and S. K. Scott, "Oscillations of an exothermic reaction in a closed system. I. Approximate (exponential) representation of Arrhenius temperature-dependence", Proc. Roy. Soc. Lond. Ser. A Math. Phys. Eng. Sci. 416 (1988) 321-341.

[17] P. Gray and S. K. Scott, Chemical oscillations and instabilities: non-linear chemical kinetics (Oxford University Press, Oxford, 1990).

[18] J. Guckenheimer and P. Holmes, Nonlinear oscillations, dynamical systems, and bifurcations of vector fields, Springer Applied Mathematical Sciences 42 (Springer, New York, 2002).

[19] E. Hopf, "Abzweigung einer periodischen Lösung von einer stationären Lösung eines Differentialsystems”, Ber. Verh. Sachs. Akad. Wiss. Leipzig Math.-Nat. 94 (1942) 3-22.

[20] S. R. Kay and S. K. Scott, "Oscillations of simple exothermic reactions in a closed system. II. Exact Arrhenius kinetics”, Proc. Roy. Soc. Lond. Ser. A Math. Phys. Eng. Sci. 416 (1988) 343-359.

[21] J. H. Merkin, D. J. Needham and S. K. Scott, "Oscillatory chemical reactions in closed vessels", Proc. Roy. Soc. Lond. Ser. A Math. Phys. Eng. Sci. 406 (1986) 299-323.

[22] M. Nelson, "Mechanisms for hydrocarbon oxidation-The Sal'nikov thermokinetic oscillator", Short articles on combustion, Dec 212002 , available at: http: / /www. uow. edu . au/ mnelson/review.dir/salnikov.html.

[23] L. Perko, Differential Equations and Dynamical Systems (Springer, New York, 1991).

[24] I. Y. Sal'nikov, "A thermokinetic model of homogeneous periodic reactions", Dokl. Akad. Nauk SSSR 60 (1948) 405-408, Russian.

[25] I. Y. Sal'nikov, "Contribution to the theory of the periodic homogenous chemical reactions: II A thermokinetic self-excited oscillating model", Zh. Fiz. Khim. 23 (1949) 258-272, Russian.

[26] S. K. Scott, Chemical Chaos (Oxford Science Publications, Clarendon Press, Oxford, 1991).

[27] M. J. Sexton and L. K. Forbes, "A note on oscillation in a simple model of a chemical reaction", J. Austral. Math. Soc. Ser. B 37 (1996) 451-457.

[28] H. S. Sidhu, M. J. Sexton, M. I. Nelson, G. N. Mercer and R. O. Weber, "A simple combustion process in a semibatch reactor", in EMAC 2000 Proceedings (eds. May, Fitz-Gerald and Grundy), (Institution of Engineers, Australia, 2000) 251-254. 